\title{
On the determination of post-Newtonian parameters with BepiColombo radio science experiment
}

\author{
L. Imperi*, G. Schettino** and L. Iess* \\ *Department of Mechanical and Aerospace Engineering, \\ Sapienza University of Rome, Rome, Italy \\ E-mail: luigi.imperi@uniroma1.it \\ ** Department of Mathematics, \\ University of Pisa Pisa, Italy \\ E-mail: gschettino@dm.unipi.it
}

\begin{abstract}
One of the main goals of the Mercury Orbiter Radio science Experiment (MORE), onboard the ESA-JAXA BepiColombo mission to Mercury, is to perform a test of gravitational theories by means of high precision radio-observables, constraining several Post-Newtonian (PN) parameters. This will be performed in two steps: (i) with a superior solar conjunction experiment during the cruise phase of the mission; (ii) by reconstructing the orbit of Mercury around the Sun once the spacecraft will be arrived at Mercury. In this work we present the results of numerical simulations of the MORE relativity experiment, carried out in a realistic scenario, showing how the experiment can improve over current estimates.
\end{abstract}

Keywords: BepiColombo; mercury; general relativity; superior conjunction.

\section{The relativity experiment of BepiColombo}

BepiColombo is a joint mission between the European Space Agency (ESA) and the Japanese Aerospace eXploration Agency (JAXA), devoted to the exploration of Mercury [1]. It is composed by two spacecraft: the Mercury Magnetosphere Orbiter (MMO), provided by JAXA, investigating the exosphere and the magnetosphere of the planet, and the Mercury Planetary Orbiter (MPO), provided by ESA, equipped with a suite of instruments for the analysis of the surface geology and the deep interior.

The Mercury Orbiter Radio science Experiment (MORE), one of the experiments onboard the MPO, will map the hermean gravity field and carry out tests of relativistic gravity. It consists of ground and onboard instrumentation enabling a highly stable, multi-frequency radio link in X and Ka bands. Two-way Doppler and range observables, obtained from this advanced radio-link, will be unaffected by plasma noise and are expected to attain accuracies of at least 3 micron/s (at 1000 seconds integration time) and $20 \mathrm{~cm}$, at nearly all solar elongation angles [5]. Furthermore, MORE will benefit from a direct measurement of the non-gravitational accelerations, provided by the Italian Spring Accelerometer (ISA).

The estimation of the gravity field and the rotational state of Mercury will be carried out by using the MPO as a test mass around the planet. Its Mercurycentric orbit will be reconstructed using primarily Doppler observables (gravimetry and rotation experiments [8], [3]). On the other hand, range observables provide information about the heliocentric motion of Mercury, thus allowing accurate fundamen- 
tal physics tests (relativity experiment [9]). Those tests are particularly sensitive because Mercury is affected more than any other planet by solar gravity and an improved determination of several Post-Newtonian (PN) parameters is expected. Among the PN parameters, $\gamma$ has the peculiarity to affect not only the motion of the planet, but also the propagation of radio-waves. Therefore a test on $\gamma$ may be performed both in the cruise phase and once that BepiColombo will be in its final orbit around Mercury.

\section{The Superior Solar Conjunction experiment during the cruise}

According to General Relativity (GR), photons are delayed (and deflected) by a gravity field. The effect, known as Shapiro time delay [13], is strongly enhanced during a Superior Solar Conjunction (SSC). In this case the approximate expression of the delay and the corresponding frequency shift for a two-way radio signal are given by $([2],[4])$ :

$$
\Delta t=2 \frac{(1+\gamma) G M}{c^{3}} \ln \left(\frac{4 R_{1} R_{2}}{b^{2}}\right) ; \frac{d \Delta t}{d t}=-4 \frac{(1+\gamma) G M}{c^{3} b} \frac{d b}{d t} ;
$$

where $G M$ is the gravitational parameter of the body producing the space-time curvature (the Sun in our case), $b$ the impact parameter (the distance of the radio beam asymptote from the body) and $c$ the velocity of light; $R_{1}$ and $R_{2}$ are respectively the positions of the transmitter and receiver in a system whose origin is in the center of mass of the body. The value of $\gamma$, which measures the space curvature induced by unit mass, is unity in GR. Since the effects in Equations (1) affect both range and Doppler measurements, a SSC is an opportunity to test GR determining the PN parameter $\gamma$. This kind of experiment was already performed in 2002 by the Cassini-Huygens mission during its cruise phase to Saturn; in that case $\gamma$ was estimated to be $\left(1+(2.1 \pm 2.3) \times 10^{-5}\right)[2]$.

In the current scenario (to April 2016), the launch is scheduled for April 2018, with arrival at Mercury in December 2024. We report results for a previous cruise trajectory (launch in January 2017), with ten exploitable SSC offered in the cruise phase [12]. Table 1 summarizes the formal uncertainty $\sigma_{\gamma}$ in the estimation of $\gamma$ for each conjunction.

Table 1. Formal uncertainties $\sigma_{\gamma}$ in the estimation of $\gamma$ for each of the ten superior solar conjunction exploitable in the cruise phase, for a launch date in January 2017.

\begin{tabular}{ccccccccccc}
\hline SSC & I & II & III & IV & V & VI & VII & VIII & IX & X \\
\hline \hline $\boldsymbol{\sigma}_{\boldsymbol{\gamma}} \cdot \mathbf{1 0}^{-\mathbf{6}}$ & 10.4 & 7.8 & 9.2 & 6.1 & 5.1 & 6.3 & 7.9 & 5.0 & 4.3 & 6.5 \\
\hline
\end{tabular}

The adopted setup for retrieving the estimates, along with further results, are given in [6]. The SSC experiment of BepiColombo is expected to provide an estimate of $\gamma$ at level of $5 \times 10^{-6}$, with a significant improvement over the Cassini result. Similar accuracies are expected also for the new trajectory (launch in April 2018). 


\section{Constraining the PN parameters during the orbital phase}

After insertion into Mercury orbit, BepiColombo will start its nominal one year orbital phase around the planet. Testing gravitational theories requires the determination of the orbits of Mercury and the Earth with unprecedent accuracy in a fully relativistic frame. The Newtonian Lagrangian of the $N$-body problem must include some corrective terms of PN order one. MORE aims to estimate, within a global least squares fit, the following PN parameters:

- $\gamma$, which parameterizes the velocity-dependend modification of the 2-body interaction and gives information about the space-time curvature through the Shapiro effect $(\gamma=1$ in GR);

- $\beta$, related to the non-linear 3 -body general relativistic interaction $(\beta=1$ in GR);

- $\eta$, describing possible violations of the strong equivalence principle $(\eta=0$ in GR);

- $\alpha_{1}$ and $\alpha_{2}$, which describe the preferred frame effects $\left(\alpha_{1}=\alpha_{2}=0\right.$ in GR).

For $\gamma$, we have adopted the a priori value of $5 \times 10^{-6}$, coming from the simulation of the SSC experiment in the cruise phase. Additional parameters that will be estimated are the gravitational parameter $G M_{\odot}$ of the Sun, its time derivative $\zeta_{\odot}$ and the solar oblateness factor $J_{2 \odot}$.

Since the orbital effects of $\beta$ and $J_{2 \odot}$ are quite similar, they are strongly correlated [8]. The correlation is largely removed if we assume that the PN parameters are linked by means of the Nordtvedt relation [11]:

$$
\eta=4(\beta-1)-(\gamma-1)-\alpha_{1}-\frac{2}{3} \alpha_{2} .
$$

This corresponds to assuming that gravity is described by a metric theory. Moreover, the orbit determination process shows a rank deficiency of order 4 , given by an approximate symmetry with respect to the rotation group $\mathrm{SO}(3)$ applied on the orbits of Mercury and the Earth, and a further approximate symmetry for scale changes. Hence, we solve for only eight of the twelve components of the planetary state vectors, keeping fixed the position of the Earth and its velocity component orthogonal to the ecliptic plane [9].

The BepiColombo relativity experiment relies almost entirely on range observables [8]. Hence an assessment of possible systematic errors in range measurements is of paramount importance. In the present work we consider the impact of the transponder aging. Systematics effects introduced by the aging directly corrupt the range measurements, but their amount is not exactly predictable. Thanks to the onboard calibration system, it can be assumed that the uncompensated group delay of the transponder is limited to a few tens of centimeters at the end of the nominal mission. In order to test the impact on the solution, we considered a quadratic increase of internal delay up to $20 \mathrm{~cm}$. This function was added to the range observables in the simulations. In the simulation setup the PN parameters 
Table 2. $\mathrm{PN}$ and related parameters estimate $\left[G M_{\odot}\right.$ is given in $\mathrm{km}^{3} / \mathrm{s}^{2}, \zeta \odot$ in $y^{-1}, J_{2 \odot}$ is un-normalized].

\begin{tabular}{cccccc}
\hline & Formal & True error & True/Formal & Current accuracy & Reference \\
\hline \hline $\boldsymbol{G M}_{\odot}$ & $3.7 \mathrm{e}-2$ & $1.2 \mathrm{e}-1$ & 3.3 & - & {$[16]$} \\
$\boldsymbol{J}_{\mathbf{2} \odot}$ & $3.5 \mathrm{e}-10$ & $1.7 \mathrm{e}-9$ & 5.0 & - & {$[16]$} \\
$\boldsymbol{\zeta}_{\odot}$ & $1.9 \mathrm{e}-14$ & $8.5 \mathrm{e}-14$ & 4.4 & $7 \mathrm{e}-13$ & {$[10]$} \\
$\boldsymbol{\beta}$ & $3.4 \mathrm{e}-7$ & $1.4 \mathrm{e}-6$ & 4.1 & $1 \mathrm{e}-4$ & {$[15]$} \\
$\boldsymbol{\gamma}$ & $8.4 \mathrm{e}-7$ & $8.4 \mathrm{e}-7$ & 1.0 & $2 \mathrm{e}-5$ & {$[2]$} \\
$\boldsymbol{\alpha}_{\mathbf{1}}$ & $4.4 \mathrm{e}-7$ & $2.4 \mathrm{e}-6$ & 5.5 & $6 \mathrm{e}-6$ & {$[7]$} \\
$\boldsymbol{\alpha}_{\mathbf{2}}$ & $6.7 \mathrm{e}-8$ & $6.7 \mathrm{e}-8$ & 1.0 & $3 \mathrm{e}-5$ & {$[7]$} \\
$\boldsymbol{\eta}$ & $1.9 \mathrm{e}-6$ & $2.8 \mathrm{e}-6$ & 1.5 & $4 \mathrm{e}-4$ & {$[15]$} \\
\hline
\end{tabular}

are estimated together with the gravity coefficients and the rotational parameters of Mercury. However, we find that solving for the relativity parameters alone (i.e. assuming that the gravity field and rotational state are perfectly known) would not lead to significant differences in the results [14]. This means that the relativity experiment is essentially independent from the gravimetry and rotation ones.

Table 2 shows the results of the simulations. For almost all parameters the true error $^{\mathrm{a}}$ is slightly larger than the computed formal uncertainty due to the systematic error in the range observables. However, under our assumptions, each parameter is determined to an accuracy better than the current one.

\section{Conclusions and perspectives}

In this work we presented the expected results from the BepiColombo relativity experiment, using a realistic simulation scenario for the January 2017 launch trajectory. A significant improvement (to $5 \times 10^{-6}$ ) in the determination of the PostNewtonian parameter $\gamma$ is expected during the cruise phase of the mission thanks to a superior solar sonjunction experiment. Several Post-Newtonian parameters can be determined with improved accuracies by tracking the spacecraft during the orbital phase around Mercury.

We evaluated the impact on the estimate due to the systematic errors arising from a variation in time of the transponder group delay (aging), finding that the ratio of the true to formal errors may be as large as 5 for some parameters if the group delay varies by $20 \mathrm{~cm}$ over the one year orbital phase. A better assessment of the transponder aging is therefore very important.

Lastly, we underline that the effect of the uncertainties in the knowledge of the solar system dynamics must be carefully evaluated as well. Indeed, an incorrect model of the solar system would bring to deviations in the orbits of Mercury and the Earth, with an impact on the solution that should be quantified. A detailed analysis of these effects is under way.

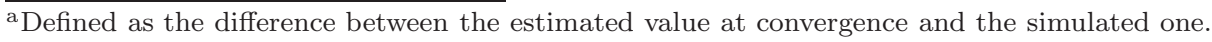




\section{References}

1. Benkhoff J. et Al., Planet. Space Sci. 58, 2 (2010).

2. Bertotti B., Iess L. \& Tortora P., Nature 425, 374 (2003).

3. Cicalò S. \& Milani A., MNRAS 427, 468 (2012).

4. Iess L. et Al., Class. Quant. Grav. 16, 1487 (1999).

5. Iess L. \& Boscagli G., Planet. Space Sci. 49, 1597 (2001).

6. Imperi L. \& Iess L., in MetroAeroSpace, IEEE, 129-134, (2015), doi:10/1109/MetroAeroSpace.2015.7180642.

7. Iorio L., Int. J. Mod. Phys. D 23, 14500006 (2014).

8. Milani A. et Al., Planet. Space Sci. 49, 1579 (2001).

9. Milani A. et Al., Phys. Rev. D 66, 082001 (2002).

10. Muller J. \& Biskupek L., Class. Quantum Grav. 24, 4533 (2007).

11. Nordtvedt K., Apj 161, 1059 (1970).

12. Jehn R., MAS working paper 525, (2014).

13. Shapiro I.I., Phys. Rev. Lett. 13, 789 (1964).

14. Schettino G., et Al., in MetroAeroSpace, IEEE, 135-139, (2015), doi:10/1109/MetroAeroSpace.2015.7180642.

15. William J.G., Turyshev S.G. \& Boggs D.H., Int. Mod. Phys. D 18, 1129 (2009).

16. The order of magnitude in the actual knowledge on $G M_{\odot}$ and $J_{2 \odot}$ may be roughly evaluated as the difference between their values in different sets of ephemerides, which is at level of respectively about few $\mathrm{km}^{3} / \mathrm{s}^{2}$ and about $5 \times 10^{-8}$ (un-normalized). 